\title{
Textural Analysis Based Classification of Digital X-ray Images for Dental Caries Diagnosis
}

\author{
Geetha $\mathrm{V}^{\mathrm{a}^{*}}$, Aprameya K. $\mathrm{S}^{\mathrm{b}}$ \\ ${ }^{a}$ Associate Professor, Department of Electronics and Communication Engineering, University BDT College of \\ Engineering, Davanagere - 577004, Karnataka, India. \\ ${ }^{b}$ Associate Professor, Department of Electrical and Electronics Engineering, University BDT \\ College of Engineering, Davanagere - 577004, Karnataka, India.
}

Received: 13 January 2019; Accepted: 25 April 2019; Published: 08 May 2019

\begin{abstract}
In this paper, we propose a suitable textural feature for diagnosis of dental caries in digital radiographs. The dental diagnosis system consists of Laplacian filter for image sharpening, adaptive threshold and morphological operations for segmentation, and support vector machine (SVM) as a classifier. In segmented image, textural features are extracted, and applied to the classifier, to classify the image as caries or normal. Experimental results indicate that GLCM (Grey Level Co-occurrence Matrix) and GLDM (Grey Level Difference Method) textural features are giving better performance measures as compared to other types of textural features with an accuracy of $96.88 \%$, sensitivity of 1 , specificity of 0.8667 and precision of $96.08 \%$. The data were analyzed by Analysis of Variance (ANOVA), at a significant level of 5\%. This result indicates that the interaction of feature extraction methods on performance measures are significant. Hence, GLCM or GLDM features provide reliable decision support for dental caries diagnosis.
\end{abstract}

Index Terms: Dental radiography, computer aided diagnosis, dental caries, textural analysis.

(C) 2019 Published by MECS Publisher. Selection and/or peer review under responsibility of the Research Association of Modern Education and Computer Science.

\section{Introduction}

Dental caries or tooth decay causes bacterial damage to teeth and is preventable if detected at an early stage. If caries touches pulp region, the treatment become complicated. Many caries detection methods available, are based on either visual examination or tactile sensation, some of them have low sensitivity, and some are very

* Corresponding author. Tel.: 919480226255

E-mail address: gvespdvg@gmail.com 
expensive [1]. Because of the difficulties in detecting caries at an early stage, clinical examination needs to be supplemented with radiographic examination [2]. Radiography uses relatively high dose of ionized radiations that are injurious to health [1]. Dental radiographs or digital dental X-ray images are convenient for computer aided dental X-ray diagnosis. These images use relatively low level of radiation exposure and makes image enhancement possible. Hence, computer assisted dental diagnosis greatly helps in caries identification.

Caries are in various shape and size. It is an irreversible microbial disease affects calcified tissues of the teeth. Proper treatment given at earlier stage helps to avoid further tooth damage. Caries located in between teeth are invisible to human eye. Use of digital radiography helps in diagnosis of caries which are in between teeth, at an early stage. Under uneven exposure, as the intensities of teeth and bone area are alike, it is difficult to distinguish between tooth and bone areas. Due to lack of clear demarcation between region of interest and due to arbitrary teeth variation, manual $\mathrm{X}$-ray image analysis is a challenging problem [3].

Human inspection may vary from dentist to dentist, and, as such, does not give a quantitative measurement. There is a need for an effective methodology to find dental caries. Various techniques that are already available are either very expensive machinery or with low specificity. Currently no system exist that are user friendly, simple, cost effective caries detection system, that can provide easily understandable information about the detection and assessment of dental caries. All these issues indicate a need for an effective low-cost methodology to find dental caries, that allows dental professionals to interpret data quickly and reliably.

It is necessary to implement an efficient, fully automatic and accurate algorithm for detection of dental caries. And, evaluation of severity of caries and location of caries affected area is necessary for giving proper treatment. This study is to develop an algorithm for an automatic computer-based diagnosis of dental caries in dental radiographs using image processing techniques.

\section{Related Work}

Very low radiation dosage used in dental radiography results in very noisy, low contrast and dark x-ray images. The image quality can be improved by image enhancement techniques. Enhancement filters improve the diagnostic performance of digital radiograph images [4]. Further, teeth segmentation performance can be improved by image enhancement method [5]. In previous work, it has been found that contrast stretching, morphological operations, PLT, homomorphic filters, CLAHE and gaussian filters are used for enhancement of dental caries. Manuela DFB et al., has given opinion to use sharpen filter to improve the diagnostic performance of dental caries [6]. Laplacian filter uses second derivative to detect the strong features such as edges of the tooth region [7]. Segmentation of teeth is a significant problem due to teeth variation in shape and size, arrangement of teeth varies between one person to another [8]. In dental radiographs there are variations from one image to another due to variation in exposure. Variations from one region to another with in the image makes the segmentation very challenging. The performance of a segmentation method is affected by noise embedded in images [3]. Due to substantial noise present in dental radiograph images, the edge detection method of segmentation does not give consistent result for all the images in the database. There is hardly any segmentation method that performs well in all problems [9].

Since intensity of bone area is very close to intensity of teeth, it is difficult to use single threshold for the whole image. Hence Nomir et al. used iterative thresholding followed by adaptive thresholding to segment the teeth from bone area and background in human identification system using dental X-ray images [10]. SenthilKumaran presents a method of edge detection of dental X-ray image using artificial neural network approach [11]. The same author has given another method for detection in dental radiographs using Genetic algorithm [12].

Feature extraction is the process of analysing the texture of an image. Texture is a repeated pattern of local variation in image intensity [13]. GLCM is one of the popular methods of estimating textural information present in the images. The features extracted using GLCM are used for object recognition system in dental works $[14,15,16]$. Ainas A. ALbahbah et al. used inter pixel autocorrelation coefficient as input features for tooth caries detection [17]. Wei Li et al. used HOG descriptors, autocorrelation coefficient and GLCM as 
feature parameters for detection of tooth decay diagnosis using back propagation neural network (BPNN) $[18,19]$. Shou Li et al. used eigen patch, a window-based feature vector, and SVM classifier for dental aided lesion detection in dental X-ray images [20]. Using the same segmentation, feature extraction and classifier, Shou $\mathrm{Li}$ et al. developed an automatic method of variational level set segmentation-based computer aided dental X-rays analysis which works in clinical environment [21]. Ingrid Nurtanio et al. uses first order statistical textural features, second order features using GLCM and higher order statistical features using GLRLM (Grey Level Run Length Matrix) for classification of cyst and tumour lesion in dental panoramic images [22]. Farzana Shahar Banu uses GLCM features for classification of dental cysts in dental radiographs [23]. Raju et al. proposed a method of feature extraction of dental x-ray radiograph based on shape and GLCM texture of extracted tooth for human identification purpose [24].

Opera et al. implemented an algorithm using object-oriented image processing software for detection of caries. In this work, dental X-ray image is segmented into individual teeth, followed by binarization and edge detection operation to get outline of the dental caries [25]. Qaramaleki et al. proposed a technique for secondary caries diagnosis in digital radiographs with $82 \%$ accuracy. In this work, adaptive gamma correction is used for image enhancement and cumulative histogram diagram for threshold selection [26]. Many previous works are on detection of caries in colour images, but the main disadvantage of these methods is that caries in between the teeth and secondary caries are invisible in colour images [27,28]. Jufriadif et al. developed an algorithm for proximal caries detection in dental X-ray images using morphological operations [29]. Solmaz Valizadeh et al developed a computer software for Detection of Approximal Caries in Posterior Teeth using digital radiographs. [30]. Shubhangi et al. developed an algorithm to detect enamel caries and interproximal caries in dental digital radiographs [31]. Receiver operating characteristic (ROC) curve is a graph of true positive fraction (TPF) versus false positive fraction (FRP). The area under the curve (AUC) of ROC characteristics can be used as a criterion to measure performance of proposed method [32].

This paper presents a comparative study of eight different methods of features extraction for diagnosis of dental caries in digital radiographs. The method uses Laplacian filter for sharpening, segmentation stage using adaptive thresholding and morphological processing, textural feature extraction and SVM classifier for dental caries recognition $[33,34,35]$. The objective of this study is to select suitable textural features for dental caries diagnosis.

\section{Methodology}

In this paper, 8 different methods of feature extraction for diagnosis of dental caries are discussed, using Laplacian filter and SVM Classifier. Some of these methods uses adaptive thresholding and morphological processing for image segmentation. All experiments are conducted in MATLAB version R2017a, running on a laptop, Intel core i7, 7th Generation with 4 GB RAM.

\subsection{Dataset}

The images analysed in this work are obtained from SJM Dental College, Chitradurga, Karnataka, India using Gendex X-ray machine with RVG sensor of the type Sirona. These images are taken for the purpose of periodontal, endodontic or orthodontic treatment. The database considered for this study comprises 64 digital $\mathrm{X}$-ray images out of which 49 are caries images and 15 are normal images. Caries in the dental X-ray images are annotated by a dentist. Train database consists of 25 caries and 10 normal digital X-ray images and test database consists of 49 caries and 15 normal digital X-ray images.

\subsection{Pre-processing}

The dental X-ray images are converted to bmp format and resized to $256 \times 256$ of class double. Differential operator given by the divergence of the gradient of a function on Euclidean space is called as Laplacian 
operator. This operator uses second derivative to detect the strong features such as edges of the tooth in digital X-ray images in all the methods discussed in this paper, Laplacian filter is used for image sharpening. The resized image is sharpened using Laplacian filter to extract the intensity variation information of the image objects present in dental X-ray images.

\subsection{Segmentation}

Laplacian filter fails to provide edges of teeth having the same intensity as the background. To overcome this problem, segmentation is necessary. The idea behind adaptive thresholding is that smaller regions in an image have approximately uniform illumination. Hence, the median value of each sub image is taken as optimum threshold to partition the sharpened image into multiple subregions. The resultant image is applied with erosion and dilation morphological operations to get the segmented image. Segmentation stage is not used in methods 1 , 5, 6, 7 and 8. Method 2 and 3 use adaptive thresholding and morphological operations for segmentation but method 4 uses only adaptive thresholding.

\subsection{Feature Extraction}

The caries identification system tested with 8 different types of textural feature extraction (FE) methods, is mentioned in Table 1. The different textural features considered are GLDM, Local Binary Pattern (LBP) Gabor, discrete wavelet transform (DWT) and statistical and region features.

Table 1. Different Features and Methodology used for Dental Caries Diagnosis

\begin{tabular}{|c|c|c|c|}
\hline Methods & $\begin{array}{l}\text { Type of } \\
\text { features }\end{array}$ & $\begin{array}{l}\text { No. of } \\
\text { features }\end{array}$ & Methodology used \\
\hline 1 & Gabor & 163840 & Laplacian + Gabor FE+SVM \\
\hline 2 & Statistical & 16 & $\begin{array}{l}\text { Laplacian + Adaptive thresholding + Morphological Processing + Statistical \& region } \\
\text { properties FE + SVM }\end{array}$ \\
\hline 3 & GLCM & 22 & Laplacian + Adaptive thresholding + Morphological Processing + GLCM FE + SVM \\
\hline 4 & GLDM & 100 & Laplacian + Adaptive thresholding + GLDM FE + SVM \\
\hline 5 & LBP & 10 & Laplacian + LBP FE + SVM \\
\hline 6 & GLDM & 100 & GLDM FE + SVM \\
\hline 7 & DWT & 4 & $\begin{array}{l}\text { Laplacian + DWT FE + PCA + } 4 \text { statistical features FE (contrast, correlation, energy and } \\
\text { homogeneity) +SVM }\end{array}$ \\
\hline 8 & DWT & 5624 & Laplacian + DWT FE + PCA +SVM \\
\hline
\end{tabular}

Gabor filter is a linear filter used for texture analysis, extracts frequency content in the specific orientation in the local region of the image. In method 1, 163840 Gabor features in the Laplacian image are extracted and is directly applied to SVM, for caries detection. The features extracted in method 2 are contrast, correlation energy, Homogeneity, mean, standard deviation, entropy, Root Mean Square (RMS), variance, smoothness, kurtosis, skewness, Inverse Difference Moment (IDM), Area, centroid and bounding box. In Method 3, The 22 textural features of the segmented image are extracted using GLCM. The GLCM features are computed based on statistical distribution of intensity of pixels at a given point in an image relative to other pixels. GLCM computes the second order statistical textural measures based on relationship between of two neighbouring pixels in the segmented image, that can be used to analyse images. In methods 4 and 6, GLDM features are 
used but method 4 uses only adaptive thresholding for segmentation but no segmentation stage in method 6. In methods 7 and 8, discrete wavelet features extracted directly from dental radiograph, and these are reduced to lower dimension using Principal Component Analysis (PCA).

\subsection{Classification}

SVM uses hyper-planes to define decision boundaries to separate different classes data points. It maps the input space data points into high dimensional feature space using suitable kernel, to simplify the classification problem. Then an optimal hyper-plane separating the two classes is chosen. In all the methods discussed in this paper, SVM is used for classification. It is used for binary classification. Textural features are extracted using GLCM, are applied to SVM classifier to categorize the images as caries or normal. In this research, linear kernel is used for separating the two classes.

Fig. 1. shows the proposed architecture for dental caries diagnosis. It has two stages: training and testing stage. Digital radiographic images of the training set are passed through Laplacian filter and then segmented using adaptive thresholding and morphological processing, features of the training images are extracted using GLCM stored in the database. In the test stage, to detect the presence of the caries in the test image, it is sharpened using Laplacian filter and segmented using adaptive thresholding, and textural features are extracted, and applied to SVM classifier. Using database information, the classifier identifies the given test image as caries or normal image. Table 1 gives proposed algorithm.

Digital Radiographs

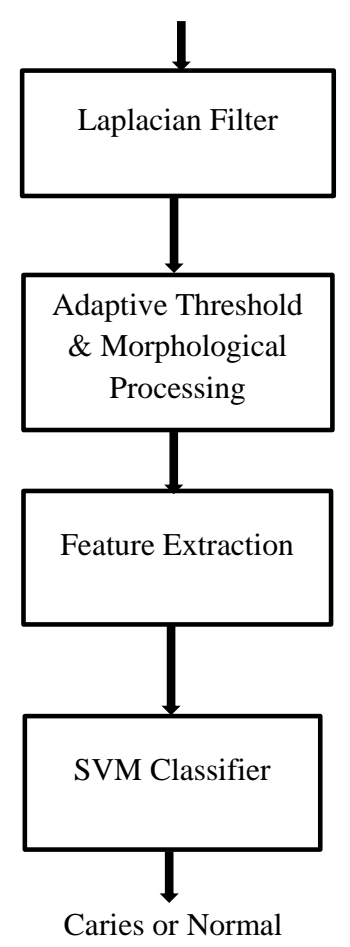

Fig.1. Proposed Caries Detection Methodology 
Table 2. Proposed Algorithm

No.
1
2
3
4
5
6
7
8
9
10
11

\author{
Resize the image to 256 X 256 of class double \\ Apply 3-by-3 Laplacian filter to get sharpened image \\ Resize the sharpened image to $100 \times 100$ of class double image 1 \\ Compute fsize $=$ fix $($ length(image1)/20) where fix rounds the value to the nearest integer towards zero. \\ Apply gaussian low pass filter of size $6 *$ fsize with standard deviation fsize for image 1 to get image 2 \\ Apply threshold to image 2 with threshold $=$ image $2 *(1-t / 100)$ to get image 3 where $t=15$ \\ Apply dilation to image 3 using structural element $b=\left[\begin{array}{llllllllllllllll}0 & 1 & 0 ; 1 & 1 & 1 ; 0 & 1 & 0\end{array}\right]$ to get image 4 \\ Apply erosion to image 3 using structural element $b=\left[\begin{array}{llllllll}0 & 1 & 0 ; 1 & 1 & 1 ; 0 & 1 & 0\end{array}\right]$ to get image 5 \\ Compute segmented_image $=$ image $4-$ image 5 \\ Extract textural features from segmented_image \\ Apply textural features to SVM classifier to classify the image as normal or caries
}

Steps

\title{
3.6. Validation
}

Dentist opinion for the true presence/absence of caries in 64 bmp images are used as a reference for estimating absolute performance numbers (true positive, true negative, true false positive and false negative). In this research, accuracy, specificity, sensitivity, precision and AUC of ROC curve of the caries detection system are computed. The validity of the results tested using a statistical analysis, two-way ANOVA at significant level of $5 \%$.

\section{Results and Discussion}

Out of 64 dental X-ray images considered, dentist identified caries in 49 radiographs. 25 caries and 10 normal X-ray images are taken for training and 49 caries and 15 normal X-ray images are considered for testing. Fig.2(a) shows the original dental X-ray image, and Fig.2(b) shows the sharpened image using Laplacian filter. It is observed that, DWT features extracted segmented images degrades the performance of the classifier. Hence any segmentation operations are not used in methods 7 and 8 . It is found that segmentation operation degrades the caries diagnosis system performance in methods 1 and 5. It is also observed that combining more than one type of these features degrades the accuracy of the diagnosis system.

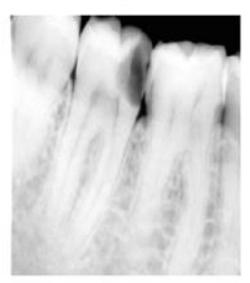

(a)

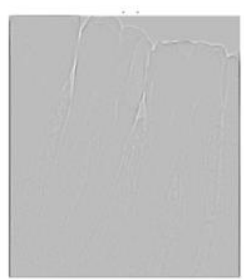

(b)

Fig.2. (a) Original Image (b) Enhanced Image using Laplacian Filter 
In order to validate feature extraction methods, the images in the database are applied to a caries identification system and performance measures accuracy, sensitivity, specificity, precision, Fscore and AUC are computed. Performance measures of the eight different methods are shown in Table 3. Accuracy, specificity and Fscore are of higher value for methods 3 and 5. But sensitivity, precision and AUC are of higher value for method 2 as compared to other methods. GLDM feature are used in methods 4 and 6 . The results shown for these methods indicates that accuracy degrades with segmentation. Classification accuracy using DWT features is better for method 8 than method 7, indicates that DWT features extracted directly from the classifier improves the performance. Performance measures of all methods are shown in Fig 3.

Table 3. Performance Measures of Different Feature Extraction Methods used for Dental Caries Diagnosis

\begin{tabular}{|l|l|l|l|l|l|l|}
\hline Method & accuracy & sensitivity & specificity & precision & F score & AUC \\
\hline 1 & 0.9219 & 0.9388 & 0.8667 & 0.9583 & 0.9488 & 0.9027 \\
\hline 2 & 0.9375 & 0.9388 & 0.9333 & 0.9787 & 0.9583 & 0.9361 \\
\hline 3 & 0.9688 & 1 & 0.8667 & 0.9608 & 0.98 & 0.9333 \\
\hline 4 & 0.8281 & 0.8776 & 0.6667 & 0.8958 & 0.8866 & 0.7721 \\
\hline 5 & 0.9063 & 0.9184 & 0.8667 & 0.9574 & 0.9375 & 0.8921 \\
\hline 6 & 0.9688 & 1 & 0.8667 & 0.9608 & 0.98 & 0.9333 \\
\hline 7 & 0.8281 & 0.8367 & 0.8 & 0.9318 & 0.8817 & 0.8084 \\
\hline 8 & 0.9219 & 1 & 0.6667 & 0.9074 & 0.9515 & 0.8333 \\
\hline
\end{tabular}

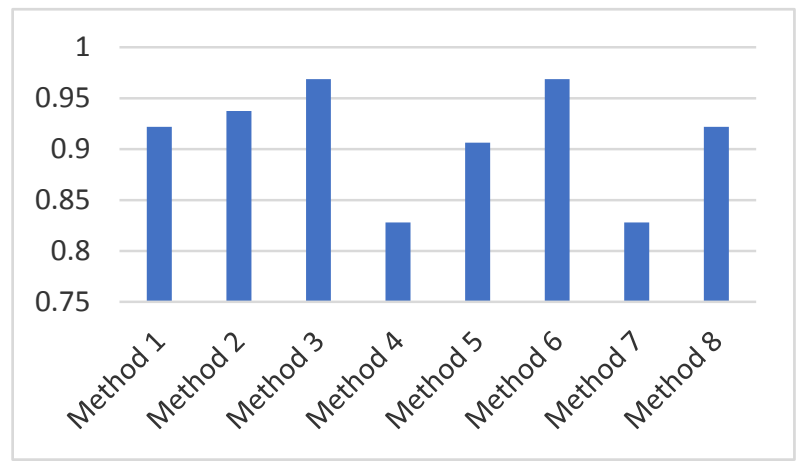

Fig.3. Comparison of Performance Parameters for the Different Feature Extraction Methods of Caries Detection

ROC curves for methods 2 and 3 are shown in Fig. 4, indicates that performance of method 2 is better than method 3 and method 5. Two-way ANOVA statistical analysis resultant significant level of $5 \%$ shown in Table 4, confirms that the interaction of the proposed method on performance parameter measures is significant. 


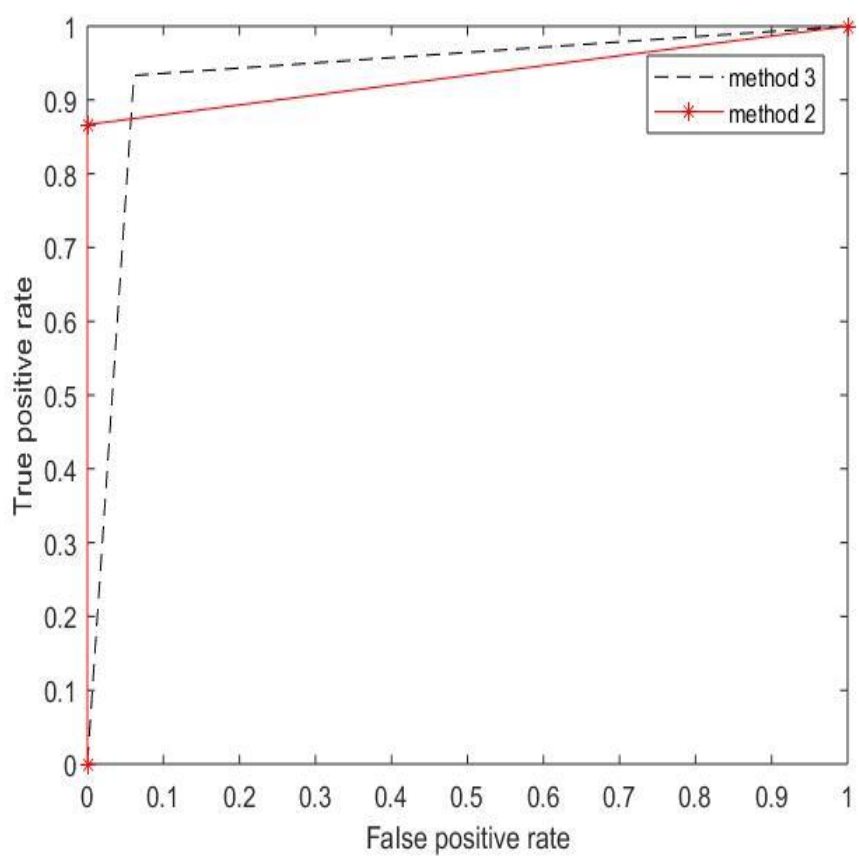

Fig.4. ROC Curve for method 2 and 3

Table 4. Two-way ANOVA Statistical Analysis Results (for data given in Table 3)

\begin{tabular}{|l|l|l|l|l|l|}
\hline Source & SS & df & MS & F & Prob > F \\
\hline Columns & 0.09293 & 4 & 0.02323 & 12.04 & 0 \\
\hline Rows & 0.07801 & 7 & 0.01114 & 5.77 & 0.0003 \\
\hline Error & 0.05403 & 20 & 0.00193 & & \\
\hline Total & 0.22498 & 39 & & & \\
\hline
\end{tabular}

\section{Conclusion}

In this paper, performance of different methods of texture based dental caries diagnostic system are discussed, in which SVM classifier is used to differentiate caries and normal images. In these system, Laplacian filter is used for enhancement, adaptive thresholding to segment the images. The experimental results show that caries and normal X-ray images could be distinguished more accurately by the diagnostic system with GLDM and GLCM features. For GLDM features, segmentation operation reduces the system accuracy. So, GLDM without segmentation (method 5) and GLCM features with adaptive thresholding and morphological processing (method 3) gives higher accuracy than other textural features for the caries diagnosis. The area under the curve observed from ROC characteristics of method 2 indicates that AUC value (0.9361) is slightly higher than methods 3 and 5. But the variation in accuracy as compared with variation in AUC between these methods, we conclude that methods 3 and 5 are better than method 2. Number of features extracted in the method 2 is only 22, but the method 5 extracts 100 features. For larger database method 5 is better than method 5 , because of less memory requirement. Hence, we can conclude that dental caries diagnosis system with Laplacian filter, adaptive thresholding, morphological processing, GLCM and SVM classifier gives more satisfied performance in detection of dental caries. 


\section{Acknowledgements}

Authors would like to thank Dr. R. Gowramma, Principal and Dr. Dharam M Hinduja, Head of the Department, Department of Conservative Dentistry and Endodontics, S.J.M. Dental College, Chitradurga for providing relevant information about dental caries and the datasets used in this research. Authors declare no conflict of interest.

\section{References}

[1] S. Datta and N. Chaki, "Detection of dental caries lesion at early stage based on image analysis technique", Proceedings of IEEE International Conference on Computer Graphics, Vision and Information Security (CGVIS), IEEE Press, pp. 89-93, 2015.

[2] B. Şenel, K. Kamburoğlu, Ö. Üçok, S. P. Yüksel, T. Özen, and H. Avsever, "Diagnostic accuracy of different imaging modalities in detection of proximal caries”, Dentomaxillofacial Radiology, Vol. 39 No.8, pp. 501-511, 2010.

[3] Anil K. Jain, Hong Chen, "Matching of dental X-ray images for human identification," Pattern Recognition, Vol. 37, pp. 1519-1532, 2004.

[4] B M. D. F. belém, G. M. B. Ambrosano, C. P. M. Tabchoury, R. I. Ferreira-Santos and F. Haiter-Neto, "Performance of digital radiography with enhancement filters for the diagnosis of proximal caries", Brazilian Oral Research, Vol. 27, No.3, pp. 245-251, 2013.

[5] Eyad Haj Said, Diaa Eldin M. Nassar, Gamal Fahmy, and Hany H. Ammar," Teeth Segmentation in Digitized Dental X-Ray Films Using Mathematical Morphology", IEEE Transactions On Information Forensics And Security, pp. 1556-6013, 2006.

[6] Manuella DFB, Glaucia MBA, Cinthia PMT, Rivea IFS and Francisco HN, "Performance of digital radiography with enhancement filters for the diagnosis of proximal caries", Oral Radiology, Braz Oral Res., Vol. 27 No. 3, pp. 245-251, 2013.

[7] B. Yousefi, H. Hakim, N. Motahir, P. Yousefi and M.M. Hosseini, "Visibility enhancement of digital dental X-ray for RCT Application Using Bayesian Classifier and Two Times Wavelet Image Fusion", Journal of American Science, Vol. 8, No.1, pp. 7-13, 2012.

[8] T. Kondo, S. Ong, and K. Foong, "Tooth segmentation of dental study models using range images", IEEE Trans. Med. Imaging, Vol. 23, pp. 350-362. 2004.

[9] Eyad Haj Said, Diaa Eldin M. Nassar, Gamal Fahmy and Hany H. Ammar, "Teeth segmentation in digitized dental x-ray films using mathematical morphology", IEEE Transactions on Information Forensics and Security, pp. 1556-6013, 2006.

[10] Omaima Nomir, M. A. Mottaleb, "A system for human identification from X-ray dental radiographs", Pattern Recognition, Vol. 38, pp. 1295-1305, 2005.

[11] N. SenthilKumaran, "Edge detection for dental x-ray image segmentation using neural network approach", The International Journal of Computer Science \& Applications (TIJCSA), Vol. 1, No.7, pp. 8-13, 2012.

[12] N. SenthilKumaran, "Genetic algorithm approach to edge detection for dental x-ray image segmentation", International Journal of Advanced Research in Computer Science and Electronics Engineering (IJARCSEE), Vol. 1, No.7, pp. 179-182, 2012.

[13] B. Vijayakumari, G. Ulaganathan, and A. Banumathi, "Dental cyst diagnosis using texture analysis", Proceedings of Machine Vision and Image Processing (MVIP), IEEE press, pp. 117-120, 2012.

[14] K. Veena Divya, A. Jatti, R. Joshi, and S. Deepu Krishna, "Characterization of dental pathologies using digital panoramic X-ray images based on texture analysis", Proceedings of 39th Annual International 
Conference of the IEEE Engineering in Medicine and Biology Society (EMBC), IEEE Press, pp. 450454, 2007.

[15] Abdolvahab Ehsani Rad, Mohd Shafry Mohd Rahim and Alireza Norouzi, "Digital dental x-ray image segmentation and feature extraction", TELKOMNIKA, Vol. 11 No. 6, pp. 3109-3114, June 2013.

[16] Tirupathi Puppala and Ramana Nagavelli, "Dental disease mobile assistant by digital image processing", International Journal of Electronics Communication and Computer Technology (IJECCT), Vol. 5, pp. 25-29, May 2015.

[17] Ainas A. ALbahbah, Hazem M. El-Bakry and Sameh Abd-Elgahany, "Detection of caries in Panoramic Dental X-ray Images using Back-Propagation Neural Network", International Journal of Electronics Communication and Computer Engineering, Vol. 7, No. 5, pp. 250-256, 2016.

[18] W Li, W. Kuang, Y. Li, Y.-J. Li, and W.-P. Ye, "Clinical X-Ray Image Based Tooth Decay Diagnosis using SVM", Proceedings of International Conference on Machine Learning and Cybernetics, IEEE Press, pp. 1616-1619, 2007.

[19] Y. Yu, Y. Li, Y. Li, J. Wang, D. Lin, and W. Ye, "Tooth Decay Diagnosis using Back Propagation Neural Network", Proceedings of 2006 International Conference on Machine Learning and Cybernetics, IEEE Press, pp. 3956-3959, 2006.

[20] Shuo Li, Thomas Fevens, Adam Krzyzak, Chao Jin and Song Li, "Semi-automatic computer aided lesion detection in dental X-rays using variational level set”, Pattern Recognition, Vol. 40, pp. 286-873, 2007.

[21] Shuo Li, Thomas Fevens, Adam Krzyzak, Song Li, "An automatic variational level set segmentation framework for computer aided dental X-rays analysis in clinical environments", Computerized Medical Imaging and Graphics, Vol. 30, pp. 65-74, 2008.

[22] Nurtanio, Ingrid; Astuti, Eha Renwi; Purnama, I. Ketut Eddy; Hariadi, Mochamad; Purnomo, Mauridhi Hery, "Classifying Cyst and Tumour Lesion Using Support Vector Machine Based on Dental Panoramic Images Texture Features", IAENG International Journal of Computer Science, Vol. 40 No.1, pp. 29-37, 2013.

[23] A. Farzana Shahar Banu, M. Kayalvizhi, Banumathi Armugam and Ulaganatham Gurenathan, "Texture based classification of dental cysts", Proceedings of 2014 International Conference on Control, Instrumentation, Communication and Computational Technologies (ICCICCT), pp. 1248-1252, 2014.

[24] J. Raju and C. K. Modi, "A Proposed Feature Extraction Technique for Dental X-Ray Images Based on Multiple Features", Proceedings of. 2011 International Conference on Communication Systems and Network Technologies, IEEE Press, pp. 545-549, 2011.

[25] Ş. Oprea, C. Marinescu, I. Liţă, M. Jurianu, D. A. Vişan, I. B. Cioc, "Image Processing Techniques used for Dental X-ray Image Analysis", Proceedings of Electronics Technology, pp. 125-129, 2008.

[26] A. Sadeghi Qaramaleki, H. Hassan pour, "A New Method to Secondary Caries Detection in Restored Teeth", International Journal of Scientific \& Engineering Research, Vol. 2, pp. 1-5, 2011.

[27] Grace F. Olsen, Susan S. Brilliant, David Primeaux, and Kayvan Najarian, "An Image-Processing Enabled Dental Caries Detection System", Proceedings of Complex Medical Engineering, IEEE Press, pp. 1-8, 2009.

[28] Adhar Vashishth, Bipan Kaushal, Abhishek Srivastava, "Caries Detection Technique for Radiographic and Intra Oral Camera Images," International Journal of Soft Computing and Engineering (IJSCE), Vol. 4, pp. 180-182, May 2014.

[29] Jufriadif Na`am, Johan Harlan, Sarifuddin Madenda, Eri Prasetio Wibowo, "Identification of The Proximal Caries of Dental X-Ray Image with Multiple Morphology Gradient Method", International Journal on Advanced Science, Engineering Technology, Vol.6, No.3, 2016.

[30] Solmaz Valizadeh, Mostafa Goodini, Sara Ehsani, Hadis Mohseni, Fateme Azimi, and Hooman Bakhshandeh, "Designing of a Computer Software for Detection of Approximal Caries in Posterior Teeth", Iran J Radiol., Vol. 12, 2015.

[31] Shubhangi Vinayak Tikhe, Anjali Milind Naik, Sadashiv D. Bhide, T. Saravanan, K. P. Kaliyamurthiie, 
“Algorithm to Identify Enamel Caries and Interproximal Caries Using Dental Digital Radiographs", Proceedings of 2016 IEEE 6th International Conference on Advanced Computing, pp. 225-228, 2016.

[32] T.A. Lasko, J.G. Bhagwat, K. H. Zou and L. Ohno-Machado, "The use of receiver operating characteristic curves in biomedical informatics", Journal of Biomedical Informatics, Vol. 38, No.5, pp. 404-415, Oct. 2005.

[33] R.M. Haralick, K. Shanmugam and I Dinstein, "Textural Features for classification", IEEE Transactions on Systems, Man and Cybernetics, Vol. 3, No.6. pp. 610- 621, Nov.1973.

[34] R.C. Gonzales, R.E. Woods S.L. Eddins, Digital Image Processing Using MATLAB, New York: Pearson Prentice Hall, 2004.

[35] J. Luts, F. Ojeda, R. Van de Plas, B. De Moor, S. Van Huffel and J.A.K. Suykens, "A tutorial on support vector machine-based methods for classification problems in chemometric," Anal. Chim. Acta, Vol. 665, No.2, pp. 129-145, 2010.

\section{Authors' Profiles}

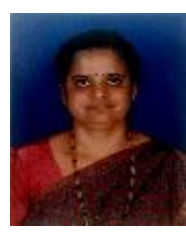

Geetha $\mathbf{V}$ received her B.E. degree from Mysore University in Electronics and Communication Engineering in 1988 and M.Tech. degree from National Institute of Technology, Calicut in Power Electronics in 1996. She is doing research for her Ph.D. degree in Department of Electrical and Electronics Engineering, Visveswaraya Technological University, Belagavi. She is a life member of ISTE, IETE. Her research interest includes Computer Vision and Neural Networks.

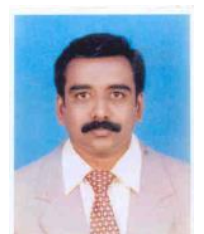

Dr. K.S. Aprameya obtained his BE degree in Electrical and Electronics Engineering in 1989, M.Tech. degree in Digital Electronics and Communication Systems in 1993 from University of Mysore and awarded Ph.D. degree in 2009 from Indian Institute of Technology, Roorkee. He is a life member for ISTE, New Delhi, MIE for "The Institution of Engineers (India)" and a member of Ultrasonic Society of India, National Physical Laboratory, New Delhi. He worked as Honorary Secretary for The Institution of Engineers (India) Davanagere Local Centre, Karnataka, India (2012-14). He is interested in image processing and its applications and ultrasonic instrumentation systems.

How to cite this paper: Geetha V, Aprameya K. S,"Textural Analysis Based Classification of Digital X-ray Images for Dental Caries Diagnosis", International Journal of Engineering and Manufacturing(IJEM), Vol.9, No.3, pp.44-54, 2019.DOI: 10.5815/ijem.2019.03.04 